ESAIM: PROCEEDINGS AND SURVEYS, December 2020, Vol. 69, p. 56-69

Philippe Helluy, Jean-Marc Hérard, Nicolas Seguin

\title{
A REMARK ON MEMORY EFFECTS IN CONSTRAINED FLUID SYSTEMS
}

\author{
Charlotte Perrin ${ }^{1}$
}

\begin{abstract}
The goal of this note is to put into perspective the recent results obtained on memory effects in partially congested fluid systems of Euler or Navier-Stokes type with former studies on free boundary obstacle problems and Hele-Shaw equations. In particular, we relate the notion of adhesion potential initially introduced in the context of dense suspension flows with the one of Baiocchi variable used in the analysis of free boundary problems.
\end{abstract}

\section{INTRODUCTION}

The general topic of this note is the mathematical modeling and analysis of partially congested flows, namely compressible flows submitted to the maximal density threshold

$$
0 \leq \rho \leq 1
$$

From the application standpoint, such constraint arises naturally in the modeling of collective motion or mixtures, in that latter case the density $\rho$ refers to the volume fraction of one phase. A common denominator of the free-congested fluid models is the continuity equation

$$
\partial_{t} \rho+\operatorname{div}(\rho u)=0
$$

where $u$ is the velocity of the flow. In the unconstrained case with no density constraint, the velocity would be defined as a desired velocity (the velocity of an agent if alone in the case of collective motion), or be prescribed by Darcy's law (flows in porous media), or even evolves according to the principle of conservation of momentum (Euler or Navier-Stokes equations). To fit with the maximal density constraint, the velocity $u$ needs however to be such that

$$
\operatorname{div} u \geq 0 \quad \text { where } \quad \rho=1 .
$$

Associated to this constraint on the divergence of the velocity field, it is common to introduce a Lagrange multiplier $p$ which satisfies, together with $\rho$, the unilateral constraint (or exclusion constraint)

$$
0 \leq \rho \leq 1, \quad p \geq 0, \quad(1-\rho) p=0 .
$$

The last equality expresses the activation of $p$ in the congested (or saturated) domain $\{\rho=1\}$, while in the free domain where $\rho<1, p=0$. Gathering these equations, we obtain a fluid model describing both the free

${ }^{1}$ Aix Marseille Univ, CNRS, Centrale Marseille, I2M, Marseille, France; e-mail: charlotte.perrin@univ-amu.fr

(C) EDP Sciences, SMAI 2020

This is an Open Access article distributed under the terms of the Creative Commons Attribution License (http://creativecommons.org/licenses/by/4.0), which permits unrestricted use, distribution, and reproduction in any medium, provided the original work is properly cited.

Article published online by EDP Sciences and available at nttps://WwW.esalm-proc.org or nttps://dol.0rg/10.1051/proc/202069056 
phases where $\rho<1$ and the congested phases where $\rho=1$. In the context of Navier-Stokes (or Euler if $\mathbb{S}=0$ ) dynamics, the system takes the form

$$
\left\{\begin{array}{c}
\partial_{t} \rho+\operatorname{div}(\rho u)=0 \\
\partial_{t}(\rho u)+\operatorname{div}(\rho u \otimes u)+\nabla p-\operatorname{div} \mathbb{S}=\rho f \\
0 \leq \rho \leq 1, \quad p \geq 0, \quad(1-\rho) p=0
\end{array}\right.
$$

where $\mathbb{S}=\mathbb{S}(\nabla u, \rho, p)$ denotes the viscous stress tensor depending on $\nabla u$, but possibly also on the density and the pressure $p$. Mathematical results on such free-congested Euler/Navier-Stokes systems are reviewed in the previous article [23. In the context of porous media, the velocity is directly linked to pressure gradient through the Darcy law

$$
u=-\frac{k}{\eta} \nabla p
$$

where $k$ is the permeability of the medium and $\eta$ the dynamic viscosity of the fluid. The associated free-congested system (called Hele-Shaw equations in the following) then reads

$$
\left\{\begin{array}{l}
\partial_{t} \rho=\operatorname{div}\left(\frac{k}{\eta} \rho \nabla p\right)+G \\
0 \leq \rho \leq 1, \quad p \geq 0, \quad(1-\rho) p=0
\end{array}\right.
$$

where $G$ is a possible additional reaction term. This system is really close to macroscopic equations used in the modeling of crowd motion (we refer to [19] and to the review paper [27], in that case $\eta$ and $k$ are fixed to 1). There, the source term is replaced by an advection term:

$$
\left\{\begin{array}{l}
\partial_{t} \rho+\operatorname{div}\left(\rho\left(u^{\text {free }}-\nabla p\right)\right)=0 \\
0 \leq \rho \leq 1, \quad p \geq 0, \quad(1-\rho) p=0
\end{array}\right.
$$

where $u^{\text {free }}$ is a free or spontaneous velocity, i.e. the velocity that an individual would follow if alone.

Going further in the modeling, we investigate in this paper some additional non-local in time phenomena, i.e. memory effects, that may be activated in the congested domain (see the system (3) presented in the next section). These memory effects, encoded in the so-called adhesion potential, were introduced formally for the free-congested Euler system by Lefebvre-Lepot and Maury [16] and justified from the mathematical viewpoint in the recent studies [21], 24], [4]. These results are reviewed in the previous paper [23. The present note completes 23 insofar as we shine a light on the possible connections with variational tools that have been introduced for obstacle type problems in the 70's, connections that were not developed in the previous synthesis. More precisely, we relate here the notion of adhesion potential introduced in the context of freecongested Euler equations with the so-called Baiocchi variable which is commonly used in the study of free boundary problems and especially for Hele-Shaw systems. We do not present here any original theoretical result but we are convinced that this new (up to the knowledge of the author) connection between the notion of adhesion potential and the concept of Baiocchi transform, could bring some new perspectives to the analysis of both Hele-Shaw models and constrained Euler (or Navier-Stokes) systems. We sketch in the conclusion of this note some potential avenues of research.

The note is organized as follows: in Section 1 we present the concept of adhesion potential for the freecongested Euler equations. Section 2 is devoted to obstacle type problems and their formulation in terms of variational inequalities. We introduce from a general viewpoint the Baiocchi transform which enables to reduce a certain type of free boundary problems to obstacle problems. Finally, in the last Section 3. we illustrate the usefulness of the Baiocchi variable in the Hele-Shaw setting. 


\section{Memory EFFECts in CONSTRAined Euler Systems, A BRIEF REVIEW}

We briefly review in this section the recent results obtained in [16], 24] and [4, our goal here is to highlight two aspects of the notion of adhesion potential: on the one hand the adhesion potential seen as a residual effect of singular lubrication forces; on the other hand the adhesion potential seen as a result of a projection of a free or spontaneous dynamics onto the set of admissible dynamics for the maximal density constraint. As said before, these results have been already presented in the recent synthesis [23], and we refer to it (and papers [16], 22], 24] and 4]) for the precise statements of the results and more details concerning the technical tools that are involved in the mathematical analysis.

\subsection{Suspension flows: singular bulk viscosity and adhesion potential}

The starting point of the analysis of memory effects in suspensions flows (i.e. mixtures composed by solid particles immersed in a viscous liquid) is the one-dimensional macroscopic model derived by Lefebvre-Lepot and Maury in $[16$. Their system reads

$$
\left\{\begin{array}{l}
\partial_{t} \rho+\partial_{x}(\rho u)=0 \\
\partial_{t}(\rho u)+\partial_{x}\left(\rho u^{2}\right)-\partial_{x}\left(\frac{\nu}{1-\rho} \partial_{x} u\right)=\rho f
\end{array}\right.
$$

whose unknowns are: the density $\rho$ of solid particles (which is supposed to remain between 0 and 1 if the particles are hard spheres) and the velocity of the mixture $u$. The system consists in pressureless Euler equations with an external forcing $f$ and a "dissipation" term representing the lubrication forces exerted by the viscous liquid (whose viscosity is denoted $\nu$ ) on the grains. Note that the singularity of the viscosity term plays here the role of a barrier: formally, it prevents the density to exceed the threshold value 1 .

Considering now the case where $\nu=\varepsilon$, the intensity of the lubrication forces becomes more and more negligible as $\varepsilon \rightarrow 0$. Lefebvre-Lepot and Maury formulate then the conjecture that the solutions $\left(\rho_{\varepsilon}, u_{\varepsilon}\right)$ of $(2)$ should converge towards solutions of the free-congested system

$$
\left\{\begin{array}{l}
\partial_{t} \rho+\partial_{x}(\rho u)=0 \\
\partial_{t}(\rho u)+\partial_{x}\left(\rho u^{2}\right)+\partial_{x} p=\rho f \\
\partial_{t} \gamma+u \partial_{x} \gamma=-p
\end{array}\right.
$$

where the potential $\gamma$, called adhesion potential, satisfies the complementary relation

$$
(1-\rho) \gamma=0, \quad \gamma \leq 0, \quad 0 \leq \rho \leq 1
$$

The last equation of system (3) expresses the fact that the pressure $p$ is the total time derivative of the potential $-\gamma$, while the complementary relation (or unilateral constraint) (4) says that both variables $\gamma$ and $p$ vanish outside the congested domain. In the saturated zone where they activate, the potential $\gamma$ keeps track (through the integral in time of $p$ ) of all the history of the external forcing $f$, which justifies the concept of memory effects. We emphasize the fact that these memory effects are here related to the maximal constraint that is imposed of the density: $\gamma$ captures the amount of compression that the fluid is exposed to but cannot accommodate due to the constraint $\rho \leq 1$. Illustrations of these memory effects can be found in the seminal paper of Maury 18 on an analogous microscopic system, and in 24$]$ for solutions of $(3)-(4)$.

Coming back to the singular limit $\varepsilon \rightarrow 0$, it appears that the pressure $p$ in (3) can be seen as the formal limit of the lubrication term $-\lambda_{\varepsilon}\left(\rho_{\varepsilon}\right) \partial_{x} u_{\varepsilon}$ where we have defined the singular "bulk viscosity" $\lambda_{\varepsilon}(\rho)=\varepsilon(1-\rho)^{-1}$. The conjecture is the following: where $\rho_{\varepsilon}$ tends to 1 , the divergence of the velocity field (here $\partial_{x} u_{\varepsilon}$ in $\left.1 \mathrm{D}\right)$ tends 
to 0 and compensates the possible blow up of $\lambda_{\varepsilon}\left(\rho_{\varepsilon}\right)$. At the approximate level, it is then natural to define an approximate potential $\gamma_{\varepsilon}=\gamma_{\varepsilon}(\rho)$ such that the following equation holds (at least in the sense of distributions):

$$
\partial_{t} \gamma_{\varepsilon}\left(\rho_{\varepsilon}\right)+\partial_{x}\left(\gamma_{\varepsilon}\left(\rho_{\varepsilon}\right) u_{\varepsilon}\right)=\lambda_{\varepsilon}\left(\rho_{\varepsilon}\right) \partial_{x} u_{\varepsilon}
$$

This equation is actually just a reformulation of the mass equation (using the concept of renormalized solutions) where the function $\gamma_{\varepsilon}$ is related to $\lambda_{\varepsilon}$ via the relation

$$
\lambda_{\varepsilon}(r)=-\left(r \gamma_{\varepsilon}^{\prime}(r)-\gamma_{\varepsilon}(r)\right) .
$$

Note in particular that when $\rho$ is close to 1 :

$$
\gamma_{\varepsilon}(\rho) \underset{\rho \rightarrow 1}{\sim} \varepsilon \ln \left(1-\rho_{\varepsilon}\right) .
$$

Formally, Equation (5) should tend to the last equation of system (3) as $\varepsilon \rightarrow 0$. Although the conjecture of Lefebvre-Lepot and Maury [16] has not been proved yet, the limit $\varepsilon \rightarrow 0$ is established in [4] and [21] for closely related systems including an additional viscosity term ensuring the estimates that allow to get some weak compactness on the sequence (weak) solutions $\left(\rho_{\varepsilon}, u_{\varepsilon}\right)_{\varepsilon}$.

The results presented in [4], [21] and [22] justify in particular the limit from (5] towards the adhesion potential equation in system (3):

Theorem 1.1 (Perrin [22]). Let $\Omega$ be the one-dimensional torus. Assume the relation (5) and appropriate initial data. From $\left(\rho_{\varepsilon}, u_{\varepsilon}\right)_{\varepsilon}$ solutions of the system

$$
\left\{\begin{array}{l}
\partial_{t} \rho_{\varepsilon}+\partial_{x}\left(\rho_{\varepsilon} u_{\varepsilon}\right)=0 \\
\partial_{t}\left(\rho_{\varepsilon} u_{\varepsilon}\right)+\partial_{x}\left(\rho_{\varepsilon} u_{\varepsilon}^{2}\right)-\partial_{x}\left(\lambda_{\varepsilon}\left(\rho_{\varepsilon}\right) \partial_{x} u_{\varepsilon}\right)-\mu \partial_{x}\left(\left(\rho_{\varepsilon}-\gamma_{\varepsilon}\left(\rho_{\varepsilon}\right)\right) \partial_{x} u_{\varepsilon}\right)=0
\end{array}\right.
$$

one can extract a subsequence $\left(\rho_{\varepsilon}, u_{\varepsilon},-\lambda_{\varepsilon} \partial_{x} u_{\varepsilon}, \gamma_{\varepsilon}\right)_{\varepsilon}$ converging weakly to $(\rho, u, p, \gamma)$ a weak solution of

$$
\left\{\begin{array}{l}
\partial_{t} \rho+\partial_{x}(\rho u)=0 \\
\partial_{t}(\rho u)+\partial_{x}\left(\rho u^{2}\right)+\partial_{x} p-\mu \partial_{x}\left((\rho-\gamma) \partial_{x} u\right)=0 \\
\partial_{t} \gamma+u \partial_{x} \gamma=-p \\
0 \leq \rho \leq 1, \quad(1-\rho) \gamma=0, \quad \gamma \leq 0
\end{array}\right.
$$

In 21], it is shown that the adhesion potential is actually a key tool to justify the existence of solutions to a model of incompressible flow with pressure-dependent viscosity. The potential $\gamma$, limit of $\gamma_{\varepsilon}\left(\rho_{\varepsilon}\right)$, can then be seen as an additional state variable which keeps track of the "micro-structure" of the system, i.e. how close from 1 is the approximate density $\rho_{\varepsilon}$. The singular limit $\varepsilon \rightarrow 0$ is a particular case of transition from soft congestion models (compressible models with singular pressure and/or viscosity laws) towards hard congestion models (coupling free and congested dynamics).

\subsection{Potential resulting from the projection on admissible dynamics}

The second aspect that we want to highlight on the adhesion potential is in relation with a projection process, the projection of the spontaneous (or free) velocity onto the set of admissible velocities for the constraint $\rho \leq 1$. This aspect is developed in the study [24] where a Lagrangian point of view is adopted. Considering an initial $\rho_{0} \in \mathcal{P}_{2}(\mathbb{R})$, absolutely continuous with respect to the Lebesgue measure and such that $\rho_{0}(x) \leq 1$ a.e. (here and hereafter we identify the probability measure with its Lebesgue density), the Lagrangian point of view consists in describing the dynamics by means of the transport map $X_{t} \in L_{\rho_{0}}^{2}(\mathbb{R})$ which is such that

$$
\rho_{t}=\left(X_{t}\right)_{\#} \rho_{0} .
$$


The set of admissible transport maps, i.e. the maps $X_{t}$ such that the associated $\rho_{t}$ satisfies the maximal constraint, is then a closed convex set $\bar{K}$ of $L_{\rho_{0}}^{2}(\mathbb{R})$. Given an initial data $\left(\rho^{\text {in }}=\rho_{0}, u^{\text {in }}\right)$, the study 24] shows that there exist weak Eulerian solutions to (3)-(4) which are constructed from the (Lagrangian) trajectories $t \mapsto X_{t}$ defined as the projection in $L_{\rho_{0}}^{2}(\mathbb{R})$ of the "free trajectory" (governed by the initial data and the external force $f$ ) onto the convex set $\bar{K}$ :

$$
X_{t}=\mathrm{P}_{\bar{K}}\left(X_{0}+\int_{0}^{t} U_{s}^{\text {free }}\right) \quad \text { where } \quad X_{0}=\mathrm{Id} \quad \text { and } \quad U_{t}^{\text {free }}=U^{\text {in }}+\int_{0}^{t} f\left(s, X_{s}\right) d s .
$$

Under reasonable assumptions on the external force $f\left(f \in L^{\infty}\left(\mathbb{R}_{+} ; \operatorname{Lip} \cap L^{\infty}(\mathbb{R})\right)\right), X_{t}$ is shown to be uniquely defined for all times $t$. Associated to the condition $X_{t} \in \bar{K}$, the Lagrangian velocity $U_{t}=\frac{\mathrm{X}_{t}}{\mathrm{t}}$ defined for a.e. $t$, is also constrained to belong a set of admissible velocities, namely the velocities which are constant in the congested domain. This set of admissible velocities is a subspace of $L_{\rho_{0}}^{2}(\mathbb{R})$ and one proves that $U_{t}$ is actually the orthogonal projection of the free velocity $U_{t}^{\text {free }}$ onto the subspace of admissible velocities.

We then define the Lagrangian potential $\Gamma_{t}$ which is such that

$$
\partial_{y} \Gamma_{t}=\mathrm{P}_{\mathrm{Adm}}\left(U_{t}^{\text {free }}\right)-U_{t}^{\text {free }}=U_{t}-U_{t}^{\text {free }} .
$$

Thanks to the projection operator properties, it is shown that $\Gamma_{t} \leq 0$ a.e. in $\mathbb{R}$ and $\Gamma_{t}=0$ a.e. outside the congested domain. Coming back to the Eulerian standpoint and defining for a.e. $t$ the triplet $\left(\rho_{t}, u_{t}, \gamma_{t}\right)$ such that

$$
\rho_{t}=\left(X_{t}\right)_{\#} \rho_{0}, \quad U_{t}=u_{t} \circ X_{t}, \quad \Gamma_{t}=\gamma_{t} \circ X_{t},
$$

one can check that the weak formulations of the equations (3)-(4) are satisfied. This justifies in the Lagrangian setting what was claimed in the previous paragraph: in the congested domain, the potential $\Gamma_{t}$ tracks the whole history of the action of the external force $f$ which is integrated in the free velocity $U_{t}^{\text {free }}$.

Concretely, these memory effects can be observed in the following situation illustrated numerically in 24]: consider initially two separated congested blocks and apply to the system a compressing external forcing $f$ in such a way that the blocks collide at a certain time $t_{1}^{*}$. From that collision time, the adhesion potential is activated and decreases while the external constraint is still compressing the system. We reverse at a time $t_{2}^{*}$ the action of $f$ which tends now to decompress the system. Then, the adhesion potential increases and goes back to 0 . At time $t_{3}^{*}$, when it is identically 0 , the blocks split. Hence, there is a waiting time before the start of the "decongestion" process, the time (interval between $t_{2}^{*}$ and $t_{3}^{*}$ ) necessary for the adhesion potential to go back to 0 .

Remark 1.2. (Case with no external force, recovering of the initial constrained Euler system) If $f=0$, one can show a retention property (i.e. the congested domain can only grow) and we guarantee the non-negativity of the time derivative of $-\Gamma$, that is the non-negativity of the pressure $P$. As a consequence, we obtain a weak solution of the initial constrained Euler system in which the equation on the adhesion potential is hidden:

$$
\left\{\begin{array}{l}
\partial_{t} \rho+\partial_{x}(\rho u)=0 \\
\partial_{t}(\rho u)+\partial_{x}\left(\rho u^{2}\right)+\partial_{x} p=0 \\
0 \leq \rho \leq 1, \quad p \geq 0, \quad(1-\rho) p=0
\end{array}\right.
$$

\section{VARIATIONAL INEQUALities AND UNILATERAL CONSTRAINTS}

The previously studied free-congested Euler system falls into the class of free boundary problems associated to an unilateral constraint ( $\mathrm{cf}$ (1)). Within this class, a certain type of free boundary problems maybe be solved via variational inequalities, directly or indirectly after an appropriate transform, the so-called Baiocchi transform introduced by Baiocchi in the 70's. The objective of this section is to recall some basic notions in 
relation with free boundary problems and variational inequalities. The interested reader is referred to [17] for an introduction to variational inequalities and to [26] (and the references therein) for details concerning the general theory on obstacle type problems.

\subsection{Canonical example: the obstacle problem}

We consider an elastic membrane, subjected to an external force $f=f(x)$ (e.g. the gravity), whose position at equilibrium is denoted $u=u(x)$ for $x \in \Omega$ and which is constrained to lie above an obstacle, i.e. $u \geq \psi$ where $\psi$ is a given function. At the boundary, we assume that $u_{\mid \partial \Omega}=g$ where $g \geq \psi_{\mid \partial \Omega}$. The function $u$ is then the unique solution of

$$
\min _{v \in K_{\psi, g}}\left\{\int_{\Omega} \frac{|\nabla v|^{2}}{2} \mathrm{x}-\int_{\Omega} f v \mathrm{x}\right\},
$$

where $K_{\psi, g}$ is formally the convex set of functions which satisfy the constraint $v \geq \psi$ and such that $v_{\mid \partial \Omega}=g$. The previous Dirichlet integral represents the elastic energy of the membrane corresponding to the graph of $v$ while the integral involving $f$ represents the work of the external force. Equivalently, it is well-known that the previous minimization problem can be rewritten under the form of the following elliptic variational inequality:

$$
u \in K_{\psi, g} \quad \text { and } \quad \int_{\Omega} \nabla u \cdot \nabla(v-u) \mathrm{x} \geq \int_{\Omega} f \cdot(v-u) \mathrm{x} \quad \forall v \in K_{\psi, g} .
$$

It can be shown that under reasonable assumptions on the data $f, g$ and $\psi$, there exists a unique solution.

Theorem 2.1 (Rodrigues 26] Chapter 4.2). Let $\Omega \subset \mathbb{R}^{n}$ be an open bounded set with Lipschitz boundary $\partial \Omega$. Let $f \in L^{p}(\Omega)$ with $p$ large enough, $g \in H^{1 / 2}(\partial \Omega), \psi \in H^{1}(\Omega)$ and $\psi \leq g$ on $\partial \Omega$. Then there exists a unique solution to the obstacle problem

$$
\begin{gathered}
u \in K_{\psi, g}=\left\{v \in H^{1}(\Omega), v \geq \psi, v=g \text { on } \partial \Omega\right\} \\
\int_{\Omega} \nabla u \cdot \nabla(v-u) x \geq \int_{\Omega} f \cdot(v-u) x \quad \forall v \in K_{\psi, g}
\end{gathered}
$$

The previous solution admits a characterization in terms of projection. Indeed, let $\tilde{g} \in H^{1}(\Omega)$ be such that $\tilde{g}_{\mid \partial \Omega}=g,\|\tilde{g}\|_{H^{1}(\Omega)} \leq C\|g\|_{H^{1 / 2}(\partial \Omega)}$, and consider the translated variables $\tilde{\psi}=\psi-\tilde{g}$, $\tilde{u}=u-\tilde{g}$. One can show that the unique solution of (7) is such that

$$
\tilde{u}=\mathrm{P}_{K_{\tilde{\psi}, 0}} l
$$

where $l$ is the unique solution (Riesz-Fréchet theorem) of

$$
\int_{\Omega} f v \mathrm{x}-\int_{\Omega} \nabla \tilde{g} \cdot \nabla v \mathrm{x}=\int_{\Omega} \nabla l \cdot \nabla v \mathrm{x}, \quad \forall v \in H_{0}^{1}(\Omega) .
$$

Let us now give some important properties satisfied by the solution $u$ which lead to the complementary equation associated to the obstacle problem.

Proposition 2.2 (Figalli [9] Section 3). The solution u of the obstacle problem (7) with zero external force $(f=0), g$ and $\psi$ of class $\mathcal{C}^{\perp}$, satisfies

$$
\begin{array}{lll}
\Delta u \leq 0 & \text { in } \quad & \Omega, \\
\Delta u=0 \quad \text { in } & \{u>\psi\} \cap \Omega .
\end{array}
$$


The previous result can be extended to non-zero external forces $f \in L^{\infty}(\Omega)$ (with optimal regularity $\mathcal{C}^{1,1}$ of the solution $u$ under a Dini continuity assumption on $f$, see for instance [5]), and we get the following complementary problem

$$
u \geq \psi, \quad-\Delta u \geq f, \quad(u-\psi)(-\Delta u-f)=0 \quad \text { a.e.. }
$$

The complementary problem has the following mechanical interpretation: either the membrane is in contact with the obstacle i.e. $u=\psi$, or the membrane lies above the obstacle $u>\psi$ and in that case the strain balances the external force, i.e. $-\Delta u=f$.

We refer to 9, 90 for further theoretical results on difficult and actual issues raised by the obstacle problem such as: the regularity of the interface, analysis of the blow up at singular points and extensions to the timeevolution case (parabolic obstacle problem). The rest of this subsection is devoted to formal rewritings of the obstacle problem.

As a consequence of the complementary problem (9), we can see the obstacle problem as a free boundary problem between the coincidence set (also called contact set) $\{u=\psi\}$ and its complement, the noncoincidence set, $\{u>\psi\}$, with an interface

$$
\Phi:=\partial\{u=\psi\} \cap \Omega=\partial\{u>\psi\} \cap \Omega
$$

which is not known a priori. If $u, \psi$ are $\mathcal{C}^{1}$ and if the domain $\{u>\psi\}$ has Lipschitz continuous boundary, the problem can be reformulated as follows:

$$
\begin{cases}-\Delta u=f & \text { in }\{u>\psi\} \\ u=g & \text { on } \partial \Omega \\ u=\psi, \quad \frac{\partial u}{\partial n}=\frac{\partial \psi}{\partial n} & \text { on } \Phi\end{cases}
$$

where $n$ denotes the exterior unit normal vector to $\{u>\psi\}$. This formulation is sometimes called "strong formulation" in the sense that we require some regularity on $\{u>\psi\}$ (that is on $\Phi$ ) and $u$ to define the normal $n$ and the derivative $\frac{\partial u}{\partial n}$. Note that we prescribe two boundary conditions at the interface $\Phi$ and one could think that the problem is a priori over-determined: one do not impose both Dirichlet and Neumann conditions for the Laplacian. Actually, this over-determination compensates the fact that the interface is unknown or, in other words, that the set $\{u=\psi\}$ is an unknown of the system.

Suppose that $u$ is a $\mathcal{C}^{1}$ solution of 10 with $\psi=0$ and assume that the interface $\Phi$ is regular and does not intersect the boundary $\partial \Omega$. For an arbitrary direction $\xi,|\xi|=1$, one can show (see 26 Section 1.3) that the function

$$
p=\nabla u \cdot \xi
$$

satisfy the free boundary elliptic problem

$$
\left\{\begin{array}{l}
-\Delta p=\nabla f \cdot \xi \quad \text { in }\{u>0\} \\
p=0, \quad \frac{\partial p}{\partial n}=-f \xi \cdot n \quad \text { on } \Phi=\partial\{u>0\}
\end{array}\right.
$$

Hence, free boundary problems 12 which are such that the gradient of the solution $p$ can be continuously extended through the interface, can be reduced to the classical obstacle problem via the inverse transformation of 11. We extend this approach in the next subsection.

\subsection{Reduction to the obstacle problem via the Baiocchi transform}

To introduce the concept of Baiocchi's transform, let us consider the following generic elliptic free boundary problem which extends 12 : in the domain $\Omega$, given the functions $k, \zeta$ and $\varphi$, we look for a function $p$ and a 
set $A \subset \Omega$ such that

$$
\left\{\begin{array}{l}
-\operatorname{div}(k \nabla p)=\varphi \quad \text { in } A \\
p=0, \quad k \frac{\partial p}{\partial n}=\zeta \cdot n \text { on } \Phi=\partial A \cap \Omega
\end{array}\right.
$$

completed by some boundary conditions at $\partial A \cap \partial \Omega$. We recall here that two boundary conditions at the interface $\Phi$ are prescribed to compensate the fact that this interface is a priori unknown. A "weaker" formulation of this problem reads

$$
\left\{\begin{array}{l}
\operatorname{supp} p \subset \bar{A} \\
-\operatorname{div}(k \nabla p)=\varphi \mathbf{1}_{A}+\zeta \cdot \nabla \mathbf{1}_{A} \quad \text { in } \mathcal{D}^{\prime}(\Omega)
\end{array}\right.
$$

To find a solution to this problem, the general idea of Baiocchi is to:

- find (pseudo-) differential operators $D_{1}$ and $D_{2}$ such that

$$
-\operatorname{div}\left(k \nabla\left(D_{1} \circ\right)\right)=(\varphi+\zeta \cdot \nabla)\left(D_{2} \circ\right)
$$

- find a regular solution $(w, A)$ such that

$$
D_{2} w=\mathbf{1}_{A}, \quad \text { with } \quad \operatorname{supp} w \subset \bar{A}
$$

One can easily check that for $D_{1,2}$ satisfying 15 and $(w, A)$ solution of 16$)$, the couple $(p, A)$, with $p=D_{1} w$, is a solution of (14). Assume that $w$ is non-negative and that the couple $(w, A)$, with $A:=\{w>0\}$, solves (16). We have then

$$
1-D_{2} w \geq 0 \text { in } \Omega \text { and } 1-D_{2} w=0 \text { in } A=\{w>0\}
$$

which means that $w$ solves the complementary problem:

$$
w \geq 0, \quad 1-D_{2} w \geq 0, \quad w\left(1-D_{2} w\right)=0,
$$

problem that can be related to a variational inequality. As a consequence, we are led to study an obstacle type problem for the variable $w$, usually called Baiocchi variable. The (integral) operator $D_{1}^{-1}$ is the Baiocchi transform. For the $2 \mathrm{~d}$ dam problem initially considered by Baiocchi in [1] (see also [26]), $D_{2}=\Delta$ and $D_{1}$ is (up to a multiplicative constant) $\partial_{y}$ the derivative with respect the vertical coordinate, so that the Baiocchi transform is the integral over the vertical. In general, the operator $D_{2}$ is not an ordinary elliptic operator and may include integro-differential terms coming from the external force $f$ and/or $\zeta$. Similarly, non-trivial boundary conditions at $\partial \Omega$ lead to difficult issues.

In this way, thanks to the Baiocchi transform, we reduce Equations [13] to an obstacle type problem, a priori easy solve, on which the issue of the regularity of the interface seems to be more accessible since $w$ should be more regular than $p$. A natural question which remains is then to know in which sense the initial free boundary problem on the variable $p$ is solved. In other words, do we ensure sufficient regularity on $w$ and $\Phi$ to define $p$ by the inverse Baiocchi transform, and give sense to Equations 13 .

\section{Application to Hele-Shaw equations}

We propose to illustrate the notion of Baiocchi variable on the system of Hele-Shaw. Initially it models a Stokes flow between two parallel flat plates, but the Hele-Shaw system was also intensively used in recent studies for the modeling of tissues growth. Here, the model will be essentially seen as the limit of the Porous Media Equation in a certain asymptotics. Other points of view could have been adopted, for instance the system can be also seen as the zero specific heat limit of the Stefan problem. In that case too, the Baiocchi transform turns out to be a crucial tool in the analysis of the model and we refer the interested reader to [28], [3].

The goal of this section is to show that the Baiocchi variable, defined as the time integral of the pressure, plays for the Hele-Shaw equations a similar role as the adhesion potential for the free-congested Euler equations. 
In particular we want to exhibit analogous phenomena as in Section 1 projection onto the set of admissible dynamics, waiting times, recording of external constraints exerted via a boundary condition or a source term, etc.

\subsection{Porous Media Equation and Hele-Shaw asymptotics}

The classical Porous Media equation reads

$$
\partial_{t} \rho=\Delta P(\rho) \quad \text { with } \quad P(\rho)=\rho^{\gamma} .
$$

This diffusion equation can be also seen as a continuity equation complemented with the Darcy law

$$
\partial_{t} \rho+\operatorname{div}(\rho u)=0 \quad \text { with } \quad u=-\frac{k_{\gamma}}{\eta_{\gamma}} \nabla p(\rho)=-\frac{\gamma}{\gamma-1} \nabla \rho^{\gamma-1} .
$$

Considering the asymptotics $\gamma=\gamma_{n} \rightarrow+\infty$, we are formally led to the limit equation

$$
\partial_{t} \rho=\Delta P
$$

where $P$ lies in the monotone graph (or Hele-Shaw graph):

$$
P= \begin{cases}0 & \text { if } 0<\rho<1 \\ \in[0,+\infty) & \text { if } \rho=1 \\ \varnothing & \text { if } \rho>1\end{cases}
$$

Equations (19)-20 form the so-called Hele-Shaw system which has to be supplemented by the initial data $\rho_{\mid t=0}=\rho_{0}$ and boundary conditions. Let us define the Baiocchi variable

$$
w(t, x)=\int_{0}^{t} P(s, x) \mathrm{s}
$$

introduced by Duvaut 7 for Stefan problems. We have formally

$$
\partial_{t}(\rho-\Delta w)=0
$$

that is

$$
\rho(t, x)=\rho_{0}(x)+\Delta w(t, x) .
$$

Since $P \geq 0$ satisfies $\sqrt{20}$, and since we have the constraint $\rho(t, x) \leq 1, w$ should solve the complementary problem (see Remark 3.1)

$$
\rho_{0}+\Delta w \leq 1, \quad w \geq 0, \quad\left(1-\rho_{0}-\Delta w\right) w=0,
$$

together with boundary conditions if the problem is set on a bounded domain $\Omega$. Hence, the Hele-Shaw system (19)-20 is naturally linked with the classical obstacle problem presented in the previous section.

Remark 3.1. The only delicate point to get 23 is actually the last equality, namely $(1-\rho) w=0$. We have used the fact that, due to 20 , we already ensure formally the same equation on $P$, i.e.

$$
(1-\rho) P=0 .
$$

Then, we invoke a retention property which states that for fixed $x$ :

$$
P\left(t_{0}, x\right)>0 \quad \Longrightarrow \quad P(t, x)>0 \quad \text { for all } t \geq t_{0} .
$$


Thanks to this property, we deduce that $P(t, x)=0$ if and only if $w(t, x)=0$, and we can replace $P$ by $w$ in (24). This retention property can be rigorously proved by using the Aronson-Bénilan inequality satisfied by the solutions of the Porous Media equation (25) and by passing to the limit $\gamma \rightarrow+\infty$. From another standpoint, the retention property says that the congested domain can only grow over time. Conversely, note that this is the growing property of the congested domain which allowed us in Remark 1.2 to guarantee the non-negativity of the pressure in the constrained Euler system (given that the adhesion potential is non-positive).

The passage to the Baiocchi variable has lead to the notion of variational solutions of the Hele-Shaw problem introduced by Eliott and Janovsky [8] in the early 80's. In [12] and 13], Gil and Quiros relate the variational solutions to weak solutions, while the link with viscosity solutions is established in [15. The latter study, not developed in the next subsections, is concerned with homogenization limit of Hele-Shaw equations with space-dependent free boundary velocities. The concept of Baiocchi variable helps there in the determination an explicit formula for the homogenized free boundary velocity.

\subsection{Effects of inconsistent initial data and boundary conditions}

Inconsistent initial data, the mesa limit. Let $n \geq 1$, and $\rho_{n}$ be a solution of the Porous Media equation

$$
\left\{\begin{array}{l}
\partial_{t} \rho_{n}=\Delta\left(\rho_{n}^{\gamma_{n}}\right) \quad \text { in } \mathbb{R}^{n} \\
\rho_{n}(0, x)=\rho_{0}(x)
\end{array}\right.
$$

As $n \rightarrow \infty$, assuming that $0 \leq \rho_{0} \leq 1$, a result of Bénilan and Crandall [2] ensures the convergence of $\left(\rho_{n}\right)_{n}$ towards the stationary profile $(\rho, P)$ given by

$$
\rho(t, x)=\rho_{0}(x) \quad \text { and } \quad P(t, x)=P_{0}(x)= \begin{cases}0 & \text { if } 0<\rho_{0}(x)<1 \\ \in[0,+\infty) & \text { if } \rho_{0}(x)=1\end{cases}
$$

We are interested here in the case where

$$
\left\|\rho_{0}\right\|_{L^{\infty}}>1
$$

initial condition which is incompatible with the limiting graph (20). Actually, it can be shown that the limit $n \rightarrow+\infty$ selects the projection of $\rho_{0}$ onto a mesa which is the "closest" compatible density profile.

Theorem 3.2 (Caffarelli, Friedman [6]). Assume that

$$
\rho_{0} \in L^{1}\left(\mathbb{R}^{n}\right) \cap L^{\infty}\left(\mathbb{R}^{n}\right)
$$

and suppose in addition that $\rho_{0}$ satisfies

$$
\rho_{0} \in \mathcal{C}^{1}\left(\mathbb{R}^{n}\right), \quad \frac{\partial \rho_{0}}{\partial r} \leq 0 \quad \text { in } \quad \mathbb{R}(r=|x|)
$$

Let $\left(\rho_{n}\right)_{n}$ be a sequence of solutions of 25. Then, as $n \rightarrow+\infty$,

$$
\rho_{n} \longrightarrow \bar{\rho} \quad \text { weakly- }{ }^{*} \text { in } \quad L_{\mathrm{loc}}^{\infty}\left(\left(t_{1}, t_{2}\right) \times \mathbb{R}^{n}\right) \quad \forall 0<t_{1}<t_{2}<+\infty \text {, }
$$

where

$$
\bar{\rho}(t, x)=\bar{\rho}(x)=\rho_{0}+\Delta \bar{w}
$$

and $\bar{w}$ is the unique solution of the obstacle problem

$$
\left(1-\rho_{0}-\Delta \bar{w}\right) \bar{w}=0, \quad \bar{w} \geq 0, \quad 1-\rho_{0}-\Delta \bar{w} \geq 0 .
$$


Observe that the limit $\bar{\rho}$ is stationary whereas the approximate density $\rho_{n}$ is not. Note in addition that the convergence takes place far from $t=0$. The behavior of $\rho_{n}$ as $t \rightarrow 0^{+}$and $n \rightarrow+\infty$ has been investigated later by Friedman and Höllig in 11. Their idea is to introduce an appropriate time rescaling and use of the Baiocchi variable to characterize the very fast transition from the initial inconsistent density $\rho_{0}$ to its projection $\bar{\rho}$. More precisely, they set

and prove that

$$
\tau=t^{\gamma_{n}}, \quad \tilde{\rho}_{n}(t, x)=\rho_{n}(\tau, x)
$$

$$
\tilde{\rho}_{n} \longrightarrow \tilde{\rho} \quad \text { weakly-* in } L_{\text {loc }}^{\infty}\left((0, \infty) \times \mathbb{R}^{n}\right)
$$

where $\tilde{\rho}(t, \cdot)=\rho_{0}+\Delta \tilde{w}(t, \cdot)$ and $\tilde{w}(t, \cdot)$ is the unique solution of the obstacle problem

$$
\left(\frac{1}{t}-\rho_{0}-\Delta \tilde{w}\right) \tilde{w}=0, \quad \tilde{w} \geq 0, \quad \frac{1}{t}-\rho_{0}-\Delta \tilde{w} \geq 0 .
$$

In other words, in the initial time boundary layer, the rescaled solution $\tilde{\rho}(t, \cdot)$ is the projection the $\rho_{0}$ into a mesa of height $1 / t$. It is important to note that while $t \leq \tilde{t}=1 /\left\|\rho_{0}\right\|_{L^{\infty}}$, the solution of the previous complementary problem 28 is $\tilde{w}=0$ and therefore $\tilde{\rho}(t, x)=\rho_{0}(x)$ for $0 \leq t \leq \tilde{t}$. Namely, one has to wait a certain amount of time (inversely proportional to $\left\|\rho_{0}\right\|_{\infty}$ ) before the collapse of the initial data begins.

Coupling with boundary conditions. Going further and considering non-trivial boundary conditions $g$ at $\partial \Omega$, the limit solution $\rho$ is no more stationary. In [13], Gil and Quiros, study the behavior of the solution $\rho_{n}$ of (18) with a boundary condition at $\partial \Omega$. They prove that

$$
\begin{aligned}
& \rho_{n}(t, \cdot) \rightarrow \rho(t, \cdot) \quad \text { strongly in } L^{1}(\Omega) \quad \text { for all } t>0 \\
& P_{n}=\rho_{n}^{\gamma_{n}} \rightarrow P \quad \text { strongly in } L^{1}\left(\left(t_{1}, t_{2}\right) \times \Omega\right) \quad \text { for all } 0<t_{1}<t_{2}
\end{aligned}
$$

where $(\rho, P)$ is solution to the Hele-Shaw problem with initial data $\bar{\rho}$ defined in $(26)$, and boundary condition $P(t, x)=g(x), x \in \partial \Omega$. Moreover, the limit $(\rho, P)$ is given by

$$
\rho(t, \cdot)=\rho_{0}(\cdot)+\Delta w(t, \cdot), \quad P=\partial_{t} w \quad \text { for } \quad t>0
$$

where the Baiocchi variable $w(t, \cdot)$ satisfies the complementary problem $(23)$ supplemented by the time dependent boundary condition

$$
w(t, x)=\operatorname{tg}(x) \quad \text { on } \partial \Omega
$$

Therefore, the whole dynamics of the problem is encoded in the obstacle problem and the boundary condition (30) satisfied by the Baiocchi variable. Note that if $\rho_{0}$ is inconsistent as in the previous paragraph, then there is again a time boundary layer and we have no more 21 but

$$
w(t, x)=w\left(0^{+}, x\right)+\int_{0}^{t} P(s, x) \mathrm{s}
$$

where $w\left(0^{+}, x\right)$, defined as the solution $\bar{w}$ of $(27)$, keeps track of the projection of the initial density $\rho_{0}$ onto a mesa.

\subsection{Effect of a growth source term}

Numerous recent mathematical studies about modeling of tissues (or tumor) growth are based on the following Hele-Shaw type equation which involves an additional source term

$$
\partial_{t} \rho=\Delta P+\rho G(P) \quad \text { with } \quad P= \begin{cases}0 & \text { if } 0<\rho<1 \\ \in[0,+\infty) & \text { if } \rho=1 \\ \varnothing & \text { if } \rho>1\end{cases}
$$


and the complementary equation

$$
P(\Delta P+G(P))=0 .
$$

In this system, the additional source term $G(P)$ models cell proliferation, proliferation which is limited by the value of the pressure $P$. Typically, $G \in \mathcal{C}^{1}$ is such that

$$
G^{\prime}<0 \text { and } G\left(P_{M}\right)=0 \text { for some } P_{M}>0
$$

The threshold pressure $P_{M}$, called homeostatic pressure, is the smallest pressure that prevents the multiplication of the cells. Under some compatibility conditions on the initial data (in particular consistent to avoid the problem of initial time boundary layer), Perthame et al. 25] proved the convergence as $n \rightarrow \infty$ of the solutions $\rho_{n}$ of the Porous Media Equation (18) with the source term $G\left(P\left(\rho_{n}\right)\right)$ towards solutions of (31) (see also [14] where a similar result is obtained for a singular approximate pressure).

Following this study, Mellet et al. analyzed further in [20] the issue of the regularity of the free boundary $\Phi(t)=\partial\{P(t, \cdot)>0\}$. Without entering precisely into the details of their study, which goes far beyond the scope of the present note, let us just explain why the Baiocchi transform is a cornerstone of [20]. The idea is to identify through the Baiocchi variable $w$ an obstacle type problem. The solution $w$ is then shown to satisfy the optimal regularity $\mathcal{C}^{1,1}$ for the obstacle problem (see references given in Section 2.1), regularity which consequently yields additional regularity on the pressure and thus on the free boundary.

Let us just identify here the relevant obstacle problem. Similarly to the transformation 21 which leads to (22)-(23), we look for a transformation that accounts for the new source term $G(P)$ and will lead to an obstacle type problem. One defines the variable

$$
w(t, x):=\int_{0}^{t} e^{-G(0) s} P(s, x) \mathrm{s} \geq 0
$$

with which equation (31) rewrites

$$
\partial_{t}\left(e^{-G(0) t} \rho-\Delta w\right)=\rho(G(P)-G(0)) e^{-G(0) t}
$$

Note that $t \mapsto \rho_{0} e^{G(0) t}$ corresponds to the "free" (with pressure $P=0$ ) evolution which is supposed to be satisfied outside the congested domain. Therefore (cf Lemma 2.4 in 20$]$ ), the following equality holds

$$
\Delta w(t, \cdot)=e^{-G(0) t} \rho(t, \cdot)-\rho_{0}(\cdot)+\int_{0}^{t} e^{-G(0) s} \rho(s, \cdot)(G(0)-G(P(s, \cdot))) \underline{s} .
$$

In the congested domain where $\rho=1$ we have then

$$
\Delta w(t, \cdot)=e^{-G(0) t}-\rho_{0}(\cdot)+\int_{0}^{t} e^{-G(0) s}(G(0)-G(P(s, \cdot))) \mathrm{s}=: F(t, x),
$$

which allows us to identify the desired obstacle problem associated with the Hele-Shaw system (31).

Theorem 3.3 (Mellet et al. 20]). For all times $t \geq 0$, the Baiocchi variable $w(t, \cdot)$ introduced in (33) is the unique solution in $H^{1}\left(\mathbb{R}^{d}\right)$ of the obstacle problem

$$
w(t) \geq 0, \quad-\Delta w(t)+F(t) \geq 0, \quad w(t)(-\Delta w(t)+F(t))=0
$$

The function $x \mapsto F(t, x)$ is then proved in $[20$ to be Dini continuous which gives the desired optimal regularity on $w(t, \cdot)$. We emphasize the fact that this the passage to the obstacle problem, that is the passage to the Baiocchi variable, which enables in 20 to characterize the dynamics and the regularity of the free boundary. 


\section{Conclusion}

We have introduced in this note two variables, the adhesion potential in the context of free-congested Euler equations, and the Baiocchi (or Duvaut) variable for Hele-Shaw equations. Despite the structural differences between the Euler equations and the Porous Media Equation, the two variables have been shown to share similar properties and to play both a crucial role in the complex dynamics of congested domains. Being both defined as the time integral of the pressure, they are activated only where $\rho=1$. In these congested regions, they keep track of the history of the external (boundary condition, external forcing) and/or internal (growth source term) compression constraint, compression that the fluid cannot accommodate due to the maximal density constraint. The activation of the two variables can be understood in both cases as the result of the projection of a "spontaneous" dynamics (dictated by the external or internal forcing) onto the set of admissible dynamics for the constraint $\rho \leq 1$. From another point of view, when free-congested systems are seen as limits of a fully compressible system (soft congestion equations with singular bulk viscosity for the free-congested Euler equations; Porous Media Equation with stiff pressure for the Hele-Shaw system), the two variables are limits of singular terms and appear to be essential in the justification of the limit process. Hence, if the apparition of memory effects in free-congested Euler systems might surprise at first sight, it turns out to be natural when, by comparison with the Hele-Shaw system, one thinks in terms of Baiocchi's transform.

Among possible perspectives based on this connection, it would be in particular interesting to characterize more precisely memory effects in the Hele-Shaw systems by forcing a "decongestion" process like what has been presented in Section 1.2 for the free-congested Euler system. On the other hand, the recent studies on Hele-Shaw problems, and in particular the one of Mellet et al. 20] presented in Section 3.3, have shown that the Baiocchi variable is appropriate when analyzing properties of the free boundary (regularity, stability etc.). This let us hope that the use of the adhesion potential could help in a similar manner in the analysis of the free boundary separating the free and congested domains in the Euler system (3). It would be another promising avenue of research which, up to our knowledge, has not been investigated yet.

\section{REFERENCES}

[1] C. Baiocchi. Su un problema di frontiera libera connesso a questioni di idraulica. Annali di matematica pura ed applicata, 92(1):107-127, 1972.

[2] P. Bénilan and M. G. Crandall. The continuous dependence on $\varphi$ of solutions of u $\mathrm{t}-\delta \varphi(\mathrm{u})=0$. Indiana University Mathematics Journal, 30(2):161-177, 1981.

[3] I. Blank, M. Korten, and C. Moore. The hele-shaw problem as a "mesa" limit of stefan problems: Existence, uniqueness, and regularity of the free boundary. Transactions of the American Mathematical Society, 361(3):1241-1268, 2009.

[4] D. Bresch, Š Nečasová, and C. Perrin. Compression Effects in Heterogeneous Media. Journal de l'Ecole Polytechnique, June 2019.

[5] L. A. Caffarelli. The obstacle problem revisited. Journal of Fourier Analysis and Applications, 4(4):383-402, 1998.

[6] L. A. Caffarelli and A. Friedman. Asymptotic behavior of solutions of $\mathrm{u} t=\delta \mathrm{u}$. Indiana University mathematics journal, 36(4):711-728, 1987.

[7] G. Duvaut. Résolution d'un problème de stefan (fusion d'un bloc de glace à zéro degré). 1973.

[8] C. M Elliott and V. Janovskỳ. A variational inequality approach to hele-shaw flow with a moving boundary. Proceedings of the Royal Society of Edinburgh Section A: Mathematics, 88(1-2):93-107, 1981.

[9] A. Figalli. Free boundary regularity in obstacle problems. Journées EDP, 2018.

[10] A. Figalli. Regularity of interfaces in phase transitions via obstacle problems. arXiv preprint arXiv:1809.02932, 2018.

[11] A. Friedman and K. Höllig. On the mesa problem. Journal of mathematical analysis and applications, 123(2):564-571, 1987.

[12] O. Gil and F. Quirós. Convergence of the porous media equation to hele-shaw. Nonlinear Analysis: Theory, Methods \&3 Applications, 44(8):1111-1131, 2001.

[13] O. Gil and F. Quirós. Boundary layer formation in the transition from the porous media equation to a hele-shaw flow. In Annales de l'IHP Analyse non linéaire, volume 20, pages 13-36, 2003.

[14] S. Hecht and N. Vauchelet. Incompressible limit of a mechanical model for tissue growth with non-overlapping constraint. Communications in mathematical sciences, 15(7):1913, 2017.

[15] I. C Kim and A. Mellet. Homogenization of a hele-shaw problem in periodic and random media. Archive for rational mechanics and analysis, 194(2):507-530, 2009. 
[16] A. Lefebvre-Lepot and B. Maury. Micro-macro modelling of an array of spheres interacting through lubrication forces. Advances in Mathematical Sciences and Applications, 21(2):535, 2011.

[17] J.-L. Lions. Quelques méthodes de résolution des problemes aux limites non linéaires. 1969.

[18] B. Maury. A gluey particle model. In ESAIM: Proceedings, volume 18, pages 133-142. EDP Sciences, 2007.

[19] B. Maury, A. Roudneff-Chupin, and F. Santambrogio. A macroscopic crowd motion model of gradient flow type. Mathematical Models and Methods in Applied Sciences, 20(10):1787-1821, 2010.

[20] A. Mellet, B. Perthame, and F. Quiros. A hele-shaw problem for tumor growth. Journal of Functional Analysis, 273(10):30613093, 2017.

[21] C. Perrin. Pressure-dependent viscosity model for granular media obtained from compressible Navier-Stokes equations. Appl. Math. Res. Express. AMRX, (2):289-333, 2016.

[22] C. Perrin. Modelling of phase transitions in one-dimensional granular flows. ESAIM: Proceedings and Surveys, 58:78-97, 2017.

[23] C. Perrin. An overview on congestion phenomena in fluid equations. Journées équations aux dérivées partielles, pages 1-34, 2018.

[24] C. Perrin and M. Westdickenberg. One-dimensional granular system with memory effects. SIAM J. Math. Anal., 50(6):59215946, 2018.

[25] B. Perthame, F. Quirós, and J. L. Vázquez. The hele-shaw asymptotics for mechanical models of tumor growth. Archive for Rational Mechanics and Analysis, 212(1):93-127, 2014.

[26] J.-F. Rodrigues. Obstacle problems in mathematical physics, volume 134. Elsevier, 1987.

[27] F. Santambrogio. Crowd motion and evolution pdes under density constraints. ESAIM. Proceedings and Surveys, $64,2018$.

[28] J. Steinbach. A variational inequality approach to free boundary problems with applications in mould filling, volume 136. Birkhäuser, 2012. 